


\title{
Unified Description of Quasi-free and Resonant Processes of Hypernuclear Production and Decay
}

\author{
R. Wünsch \\ Research Center Rossendorf Inc., PSF 19, O-8051 Dresden, Germany
}

\begin{abstract}
We review a unified description of resonant and quasi-free hypernuclear production reactions on the basis of the continuum shell-model. Both reaction mechanisms are considered as boundary cases of the same process. We apply the model to the $\left(K^{-}, \pi\right)$ and to the $\left(\pi, K^{+}\right)$reaction on light target nuclei. Particular attention is given to the consequences of the shallow hyperon-nucleus potential. Hypernuclear disintegration by baryon emission is considered as transition from the bound to the unbound part of the configurational space.
\end{abstract}




\section{Introduction}

At first glance, a hypernucleus is a strongly interacting object like an ordinary atomic nucleus, characterized by a diameter of a few femtometer and usually surrounded by electrons, which neutralize the positive charge of the nucleus. Only the quantum number strangeness $(S)$ distinguishes both kinds of nuclei. In the traditional picture, baryons are the fundamental building blocks of strongly interacting matter. While an ordinary nucleus consists of nucleons (N), a hypernucleus contains additionally, at least one hyperon (Y). Nuclear physics tries to explain the structure of a nucleus, including its excitation spectrum, transition probabilities and behaviour during nuclear reactions, on the basis of the interaction between the nuclear constituents. While ordinary nuclear physics bases on the interaction between nucleons, in hypernuclear physics hyperons are additionally involved. Due to the various selection rules for meson exchange, the hyperon-nucleon (YN) and the hyperon-hyperon (YY) interaction differ quantitatively from the nucleon-nucleon (NN) interaction. Generally, the interaction with and between hyperons is less attractive than the nucleon-nucleon interaction is. The $\Lambda N$ interaction is roughly half as strong as the $N N$ interaction, while $\Sigma N$ and $\Xi N$ interaction seems to be still weaker. Consequently, a hypernucleus is more loosely bound than an ordinary nucleus, and unbound states exhaust a larger fraction of the hypernuclear excitation spectrum.

Before studying pecularities of the YN or YY interaction we have to understood the general features of hypernuclei and the way to produce them. For that, we need both a (nonrelativistic) nuclear structure model and a reaction model describing the conversion of a nucleon, which is embedded in a nucleus, into a hyperon, e.g. by means of mesons. In both models we rely on the experience of ordinary nuclear physics. As to the nuclear model we use the nuclear shell-modell which has succesfully been applied for more than 50 years. In this model, a nuclear many-particle state is expanded within a set of basis states composed of single-particle states. A residual interaction mixes the various shell-model configurations (shell-model with configurational mixing). In the traditional version of the nuclear shellmodel (harmonic oscillator model) the single-particle states are defined by the hamiltonian of the harmonic oscillator and restricted to bound states (bound-state shell-model). Such a model does not allow the emission of nucleons or hyperons. Concentrating on the description of bound or quasi-bound nuclear states, this defect is unimportant. Since a hypernucleus is generally more loosely bound than an ordinary nucleus the produced hyperon may leave the nucleus in most cases. That is why we apply a shell-model which allows the emission of nucleons or hyperons (continuum shell-model). It describes simultaneously two hypernuclear production mechanisms which are often treated separately: the resonant (recoilless) and the quasi-free mechanism.

In the shell-model approach, all baryons are usually assumed to be stable particles, which are scattered on each other. In reality, the hyperon is unstable. This fact is ignored in our calculations. This neglect is justified for $\Lambda$ hyperons which decay only weakly. Their decay matrix-element is by several orders of magnitude smaller than the scattering matrix-element. All the heavier hyperons convert strongly into lighter ones if they are surrounded by nucleons. $Y N \rightarrow Y N$ scattering and $Y N \rightarrow Y^{\prime} N$ or $Y N \rightarrow Y^{\prime} Y^{\prime}$ convertion matrix elements are of the same order of magnitude. In this case, scattering and conversion processes should be treated on the same footing. The introduction of an imaginary part in the shell-model hamiltonian might be a possible first approximation to do that. However, there is another decay process which leads to an imaginary part in the relevant hamiltonian, since the hypernucleus itself can disintegrate and emit nucleons, hyperons or clusters of them. Both disintegration processes may interfere and must be considered carefully. We wanted to avoid the difficulties connected with the use of comlex single-particle wave functions and neglected the conversion process completely. So we had to restrict ourselves to hyperons whithout strong conversion ( $\Lambda$ hypernuclei) and to cases which seem to be only weakly affected by the conversion (loosely bound and scattering states of $\Sigma$ and $\Xi$ hypernuclei). 
Hypernuclei are usually produced by bombarding ordinary nuclei with elementary projectiles, usually mesons, of a few hundred $\mathrm{MeV}$. In this energy region, the projectile-nucleus interaction consists of a succession of projectile-nucleon interactions. The whole production cross-section can be reduced to the elementary projectile-nucleon cross-section multiplied by an effective nucleon number (impulse approximation (IA)). The elementary cross section is independent of the nuclear structure and can be taken from the experiment. The effective number contains all the nuclear and hypernuclear spectroscopic information and is our actual matter of interest. Rescattering and absorption of the projectile inside the nucleus can be described by a distorted wave-function (distorted wave impulse approximation (DWIA)).

In sect. 2, we outline the basic definitions, approximations and formulae of the continuum shell-model (CSM) and sketch the way how to determine the desired quantities numerically. In sect. 3, the CSM representation of a hypernuclear state is used to define resonance and quasi-free contributions to the production probability and to investigate the relation between both mechanisms. Their quantitative relation is evaluated in dependence on the reaction parameters. Sect. 4 is devoted to the pecularities of the shallow hyperon-nucleus potential. Hypernuclear disintegration probabilities are considered in sect. 5 . Concluding remarks are given in sect. 6 .

\section{Continuum shell-model description of bound and un- bound hypernuclear states}

In the shell-model approach, a nuclear many-particle state is expanded with respect to a single-particle basis defined by an average nuclear potential $\mathrm{u}(\mathrm{r})$. The basis states of $A$ identical particles are given by antisymmetrized products (Slater determinants) of nucleon wave-functions. For hypernuclei, the set has to be extented by hyperon states. The shellmodel can be applied to any mixture of nucleons and hyperons. The formal equations given below are independent on the number of hyperons and nucleons, respectively. Our numerical calculations, however, are restricted to hypernuclei with a single hyperon. So we can make use of the fact that there are $(A-1)$ identical partiles (nucleons) and one particle (hyperon) which is different.

In the traditional approach, the shell-model basis is restricted to bound single-particle states vanishing asymptotically for large separations of a nucleon from the center of the nucleus. Such a configurational space allows the description of bound and quasi-bound resonance states. Resonance states play an important role in many nuclear reactions including hypernuclear production. Recoilless $\Lambda$ hypernucleus production is dominated by resonance excitation and has successfully been described by bound-state shell-models (e.g. $[1,2,3])$. On the other hand, in most of the hypernuclear production reactions, the momentum transfer is larger than the Fermi momentum of the corresponding hyperon in the nucleus. In this case, the hyperon is produced in a scattering state and leaves the nucleus (quasi-free process). The shallowness of the hyperon-nucleus potential increases the quasi-free contribution additionally. Usually a hypernuclear excitation spectrum exhibits both features: a resonant part below and around the hyperon emission threshold and a quasi-free spectrum above it. Their relative weights depend on the momentum transfer and on the strength of the average hyperon-nucleus potential as well.

The continuum shell-model (CSM) allows a simultanious description of resonant and quasi-free processes on the same footing. It represents an extension of the traditional shellmodel by unbound states. Its basis functions are defined by an average baryon-nucleus potential vanishing at large separations of the baryon from the center of the nucleus. The corresponding single-particle spectrum consists of both a discrete and a continuous part. We approximate the avarage baryon-nucleus potential by a real Woods-Saxon potential 


$$
u(r)=-U_{R e} f(r)+U_{\text {s.o. }} \vec{l} \cdot \vec{\sigma} \lambda_{\pi}^{2} \frac{1}{r} \frac{d f(r)}{d r}+U_{C o u l}(r)
$$

consisting of a volume term, a spin-orbit term and, for charged particles, a Coulomb term. Its parameters are different for protons, neutrons, $\Lambda, \Sigma$ and $\Xi$ hyperons. To fit the decay thresholds we allow the nucleon parameters to be slightly state-dependend. The radial dependence $\mathrm{f}(\mathrm{r})$ of the volume term follows from the nuclear matter density usually parametrized by a Fermi distribution

$$
f(r)=\left[1+\exp \left\{\frac{r-R}{d}\right\}\right]^{-1}
$$

with an average nuclear radius

$$
R=r_{o}(A-1)^{1 / 3}
$$

in dependence of the mass number $(A-1)$ of the residual nucleus. Radius $r_{o}$ and diffuseness $\mathrm{d}$ are a little smaller for hyperons than for nucleons due to the shorter range of the hyperonnucleon interaction. The shape of the spin-orbit potential is proportional to the derivative of the Fermi distribution (2) and $\lambda_{\pi}=\hbar / m_{\pi} c$ is the Compton wavelength of the pion. The Coulomb potential for a baryon with charge number $Z$ in a nucleus with charge number $Z_{t}$ is approximated by the electrostatic potential of a homogeneously charged sphere with radius $R_{\text {Coul }}$

$$
U_{\text {Coul }}(r)=\hbar c \alpha Z Z_{t} \begin{cases}\frac{3-\left(r / R_{\text {Coul }}\right)^{2}}{2 R_{\text {Coul }}} & \text { if } r \leq R_{\text {Coul }} \\ 1 / r & \text { if } r \geq R_{\text {Coul }} .\end{cases}
$$

The charge radius $R_{\text {Coul }}$ may slightly differ from the nuclear radius $R . \alpha$ is the Sommerfeld fine-structure constant.

The nuclear hamiltonian is given in shell-model approach by

$$
\hat{H}=\hat{T}+\hat{U}+\hat{V}^{r e s},
$$

where

$$
\hat{T}=\sum_{i=1}^{A} \hat{t}_{i}
$$

is the sum of the kinetic energies $\hat{t}_{i}$ and

$$
\hat{U}=\sum_{i=1}^{A} \hat{u}_{i}
$$

the sum of the potential energies $\hat{u}_{i}$ of all A baryons. In CSM approach, it is given by eq. (1). The residual interaction

$$
\hat{V}^{r e s}=\sum_{i<k}^{A} \hat{v}_{i k}^{r e s}
$$

describes formally that part of the intranuclear baryon-baryon interaction which is not taken into account in the avarage potential. It mixes the various shell-model configurations. We approximate the residual interaction by a zero-range force with spin-exchange term

$$
\hat{\gamma}_{i k}^{r e s}\left(\vec{r}_{i}-\vec{r}_{k}\right)=-V_{o}^{r e s}\left(a+b \hat{P}_{i k}^{\sigma}\right) \delta^{(3)}\left(\vec{r}_{i}-\vec{r}_{k}\right),
$$


where $\hat{P}_{i k}^{\sigma}$ is the spin-exchange operator between particles $\mathrm{i}$ and $\mathrm{k}$. The zero-range force is an essential approximation reducing the CSM equations to a manageable level. The first two operators of the hamiltonian (5) constitute the shell-model hamiltonian

$$
\hat{H}^{o}=\hat{T}+\hat{U}
$$

and consist of a sum of single-particle operators. They determine the basis states of the shell-model. The residual interaction (8) mixes the basis states.

There are various methods of taking both resonant and quasi-free processes of hypernuclear production into account $[4,5,6,7,8]$. We use a CSM version which allows us to identify the contribution of a definite hypernuclear configurations to the total reaction probability. For that aim we define a projection operator

$$
\hat{Q}=\sum_{R}\left|\Phi_{R}\right\rangle\left\langle\Phi_{R}\right|,
$$

where $\left|\Phi_{R}\right\rangle$ is a many-particle shell-model state defined by the shell-model hamiltonian $H^{\circ}$ (10). The sum runs over all the shell-model states with all A baryons in bound single-particle states. The operator $\hat{Q}$ projects onto a subspace (Q-space) of the total many-body space. Q-space states may lie below (bound states) and above the disintegration threshold of the nucleus (resonance states). The latter are embedded in a continuum of scattering states, which starts above the first disintegration threshold. The complementary P-space is defined by a projection operator

$$
\hat{P}=1-\hat{Q} \text {. }
$$

It is made up of shell-model states with at least one baryon in the continuum of unbound states. As a further approximation we restrict the P-components to states with exactly one baryon in the continuum.

A hypernuclear state $|\Psi\rangle$ satisfies the Schrödinger equation

$$
[E-\hat{H}]|\Psi\rangle
$$

with the nuclear hamiltonian $\hat{H}$ (5). If the hypernucleus is in the ground state or in any other bound state it can completely be described by a set $\varrho$ of discrete quantum numbers. Such a state $\left|\Psi_{\varrho}\right\rangle$ has only components in the Q-subspace. Above the nuclear disintegration threshold, the excitation energy $E$ is a continuous quantum number. Let us assume that the hypernucleus may decay into two fragments: one baryon and a residual nucleus with (A-1) baryons. Other decay channels are assumed to be either closed or negligible. This approximation is in accordance with the restriction to configurations with at most one baryon in the continuum. A state decaying into two fragments is characterized by a discrete set $f$ of quantum numbers and by the continuous quantum number $E$. An unbound state is additionally characterized by its asymptotic behaviour. We use states $\left|\Psi_{E, f}^{(+)}\right\rangle$with the asymptotic of a plane wave described by quantum numbers $f$ and outgoing sperical waves in all open channels (out-asymptotic) and also states $\left|\Psi_{E, f}^{(-)}\right\rangle$with a plane wave in channel $f$ and incoming spherical waves in all open channels (in-asymptotic). An unbound state has components in either subspaces

$$
\left|\Psi_{E, f}^{( \pm)}\right\rangle=\hat{P}\left|\Psi_{E, f}^{( \pm)}\right\rangle+\hat{Q}\left|\Psi_{E, \hat{f}}^{( \pm)}\right\rangle
$$

Inserting the decomposition (14) into the Schrödinger equation (13) we get the representation [9]

$$
\left|\Psi_{Z, f}^{( \pm)}\right\rangle=\left|\xi_{E, f}^{( \pm)}\right\rangle+\left(\hat{Q}+\hat{G}_{P}^{( \pm)} \hat{H}_{P Q}\right) \frac{1}{E-\hat{H}_{Q Q}^{e f f( \pm)}} \hat{H}_{Q P}\left|\xi_{E, f}^{( \pm)}\right\rangle
$$


where we have introduced the Greenoperator $\hat{G}_{P}^{(+)}$in the P-subspace

$$
\hat{G}_{P}^{( \pm)}=\hat{P} \frac{1}{E( \pm)-\hat{H}_{P P}} \hat{P}
$$

with the P-component $\hat{H}_{P P} \equiv \hat{P} \hat{H} \hat{P}$ of the hamiltonian and the eigenstates $\left|\xi_{E, f}^{( \pm)}\right\rangle$of $\hat{H}_{P P}$

$$
\left[E-\hat{H}_{P P}\right]\left|\xi_{E, f}^{( \pm)}\right\rangle=0
$$

with the same asymptotics as $\left|\Psi_{E, f}^{( \pm)}\right\rangle$. Inserting eqs. (12) and (11) into eq. (17) one gets

$$
[E-\hat{H}]\left|\xi_{E, f}^{( \pm)}\right\rangle=-\sum_{R}\left\langle\Phi_{R}|\hat{H}| \xi_{E, f}^{( \pm)}\right\rangle\left|\Phi_{R}\right\rangle
$$

where we have used

$$
\hat{Q}\left|\xi_{E, f}^{( \pm)}\right\rangle=0
$$

following from eq. (17). The Green operator $\hat{G}_{P}^{( \pm)}$generates states with asymptotically outgoing $(t)$ and incoming (-) sperical waves, respectively. This asymptotic behaviour is ensured by adding a tiny imaginary part $\pm i \varepsilon$ to the energy $\left(E^{( \pm)}=E \pm i \varepsilon\right)$ in the denominator of the Greenoperator (16).

The effective $Q$-space hamiltonian $\hat{H}_{Q Q}^{\text {eff }}{ }^{( \pm)}$describes the coupling between the various shell-model configurations of the Q-subspace. It consists of two terms

$$
\hat{H}_{Q Q}^{e f f( \pm)}=\hat{H}_{Q Q}+\hat{H}_{Q P} \hat{G}_{P}^{( \pm)} \hat{H}_{P Q}
$$

The first term is the nuclear hamiltonian restricted to the $Q$-subspace ( $\left.\hat{H}_{Q Q} \equiv \hat{Q} \hat{H} \hat{Q}\right)$. It corresponds to the hamiltonian of the traditional bound-state shell-model and describes the configurational mixing within the Q-subspace (internal mixing). The second term causes an additional mixing of the $\mathrm{Q}$-space configurations due to the presence of the $\mathrm{P}$-space (external mixing). It produces an additional shift of the bound and resonance states and gives the resonance states a finite width. The operator

$$
\hat{H}_{P Q} \equiv \hat{P} \hat{H} \hat{Q}=\hat{P} \hat{V}^{r e s} \hat{Q}
$$

and the corresponding operator $\hat{H}_{Q P}$ mediate transitions from the $\mathrm{Q}$ - to the $\mathrm{P}$-space and vice versa. Since $\hat{H}^{\circ}$ commutes with $\hat{P}$ and $\hat{Q}$, only $\hat{V}^{\text {res }}$ gives a contribution to the transition operator. Inserting representation (11) of operator $\hat{Q}$ into eq. (15) we get

$$
\left|\Psi_{E, f}^{( \pm)}\right\rangle=\left|\xi_{E, f}^{( \pm)}\right\rangle+\sum_{R R^{\prime}}\left\langle\Phi_{R}\left|\frac{1}{E-\hat{H}_{Q Q}^{e f f( \pm)}}\right| \Phi_{R^{\prime}}\right\rangle\left\langle\Phi_{R^{\prime}}|\hat{H}| \xi_{E, f}^{( \pm)}\right\rangle\left|\Omega_{R}^{( \pm)}\right\rangle
$$

with

$$
\left.\left|\Omega_{R}^{( \pm)}=\right| \Phi_{R}\right\rangle+\left|\omega_{R}^{( \pm)}\right\rangle
$$

and

$$
\left|\omega_{R}^{( \pm)}\right\rangle=\hat{G}_{P}^{( \pm)} \hat{H}\left|\Phi_{R}\right\rangle=\hat{G}_{P}^{( \pm)} \hat{V}^{r e s}\left|\Phi_{R}\right\rangle,
$$

i.e., the state $\left|\omega_{R}^{( \pm)}\right\rangle$fulfils the equation

$$
\left[E-\hat{H}_{P P}\right]\left|\omega_{R}^{( \pm)}\right\rangle=\hat{H}_{P Q}\left|\Phi_{R}\right\rangle
$$

If one replaces $\hat{P}$ by means of eq. (12) and introduces eq. (11) one gets from eq. (25) 


$$
[E-\hat{H}]\left|\omega_{R}^{ \pm}\right\rangle=\hat{H}\left|\Phi_{R}\right\rangle-\sum_{R^{\prime}}\left[\left\langle\Phi_{R^{\prime}}|\hat{H}| \Phi_{R}\right\rangle+\left\langle\Phi_{R^{\prime}}|\hat{H}| \omega_{R}^{( \pm)}\right\rangle\right]\left|\Phi_{R^{\prime}}\right\rangle
$$

where we have used the property

$$
\hat{Q}\left|\omega_{R}^{( \pm)}\right\rangle=0
$$

following from eq. (24). According to eq. (23) the state $\left|\Omega_{R}^{( \pm)}\right\rangle$differs from the basisstate $\left|\Phi_{R}\right\rangle$ by an additional term $\left|\omega_{R}^{( \pm)}\right\rangle$describing its decay into (its generation from) the continuum of $\mathbf{P}$-space states.

Expression (22) has poles at the eigenvalues $\mathcal{E}_{\varrho}^{( \pm)}$of $\hat{H}_{Q Q}^{\text {eff }}{ }^{( \pm)}$which is energy-dependent via the Greenoperator $\hat{G}_{P}^{( \pm)}(16)$. If the excitation energy $\mathrm{E}$ is higher than the threshold of the lowest disintegration channel, $\hat{G}_{P}^{( \pm)}$is nonhermitian and thus $\hat{H}_{Q Q}^{e f f( \pm)}$ is nonhermitian too. Its eigenvalues $\mathcal{E}_{\varrho}^{( \pm)}$and eigenstates $\Phi_{\varrho}^{( \pm)}$defined by

$$
\left\langle\Phi_{\varrho}^{( \pm) *}\left|\hat{H}_{Q Q}^{\text {eff }}( \pm)\right| \Phi_{\varrho^{\prime}}^{( \pm)}\right\rangle=\mathcal{E}_{\varrho}^{( \pm)} \delta_{\varrho Q^{\prime}}
$$

are complex. Here, the signs $( \pm)$ do not refer to the asymptotic behaviour but rather indicate that $\mathcal{E}_{\varrho}^{(+)}\left(\left|\Phi_{\varrho}^{(+)}\right\rangle\right)$is eigenvalue (eigenstate) of $\hat{H}_{Q Q}^{e f f(+)}$ and $\mathcal{E}_{\varrho}^{(-)}\left(\left|\Phi_{Q}^{(-)}\right\rangle\right)$eigenvalue (eigenstate) of $\hat{H}_{Q Q}^{e f(-)}$. Both $\left|\Phi_{\varrho}^{(+)}\right\rangle$and $\left|\Phi_{\varrho}^{(-)}\right\rangle$are restricted to the $Q$-space and hence vanish asymptotically. In the case of a hermitian hamiltonian $\hat{H}$, the Greenoperator $\hat{G}_{P}^{( \pm)}$ is the only nonhermitian part of $\hat{H}_{Q Q}^{\text {eff }}{ }^{( \pm)}$and the eigenvalues and eigenvectors are comlex conjugate to each other

$$
\mathcal{E}_{\varrho}^{( \pm) *}=\mathcal{E}_{\varrho}^{(\mp)} \quad \text { and } \quad\left|\Phi_{\varrho}^{( \pm) *}\right\rangle=\left|\Phi_{\varrho}^{(\mp)}\right\rangle
$$

Real and imaginary part of the eigenvalues $\mathcal{E}_{\varrho}^{(+)}$are denoted by $E_{\varrho}$ and $-\Gamma_{\varrho} / 2$. Because of eq. (29) we have

$$
\mathcal{E}_{\varrho}^{( \pm)}=\left[E_{\varrho} \mp i \Gamma_{\varrho} / 2\right]
$$

The eigenstates $\left|\Phi_{\varrho}^{( \pm)}\right\rangle$are linear combination of the basis states $\left|\Phi_{R}\right\rangle$

$$
\left|\Phi_{\varrho}^{(+)}\right\rangle=\sum_{R} a_{\varrho}^{R}\left|\Phi_{R}\right\rangle
$$

They form another complete basis in the Q-subspace, and the $\hat{Q}$-operator can be represented by

$$
\hat{Q}=\sum_{\varrho}\left|\Phi_{\varrho}^{( \pm)}\right\rangle\left\langle\Phi_{\varrho}^{( \pm) *}\right|
$$

The coefficients $a_{\varrho}^{R}$ are generally complex and fulfil

$$
\sum_{R} a_{\varrho}^{R} a_{\varrho^{\prime}}^{R}=\delta_{\varrho \varrho^{\prime}} \quad \text { and } \quad \sum_{\varrho} a_{\varrho}^{R} a_{\varrho}^{R^{\prime}}=\delta_{R R^{\prime}}
$$

Below the lowest disintegration threshold, eigenvalues, eigenfunctions and coefficients $a_{\ell}^{R}$ are real. Using representation (32) instead of (11) one gets from eq. (15)

$$
\left|\Psi_{E, f}^{( \pm)}\right\rangle=\left|\xi_{E, f}^{( \pm)}\right\rangle+\sum_{\varrho} \frac{\left\langle\Phi_{\varrho}^{( \pm) *}|\hat{H}| \xi_{E, f}^{( \pm)}\right)}{E-E_{\mathfrak{Q}} \pm i \Gamma_{\mathscr{Q}} / 2}\left|\Omega_{\varrho}^{( \pm)}\right\rangle
$$


with

$$
\left|\Omega_{\varrho}^{( \pm)}\right\rangle=\left|\Phi_{\varrho}^{( \pm)}\right\rangle+\left|\omega_{\varrho}^{( \pm)}\right\rangle
$$

and

$$
\left|\omega_{\varrho}^{( \pm)}\right\rangle=\hat{G}_{P}^{( \pm)} \hat{H}\left|\Phi_{\varrho}^{( \pm)}\right\rangle=\hat{G}_{P}^{( \pm)} \hat{V}^{r e s}\left|\Phi_{\varrho}^{( \pm)}\right\rangle=\sum_{R} a_{\varrho}^{R}\left|\omega_{R}^{( \pm)}\right\rangle
$$

with the same coefficients $a_{\varrho}^{R}$ as in eq. (31). Equation (36) represents a decomposition of the hypernuclear state $|\Psi\rangle$ into a component $|\xi\rangle$, which is determined by the P-space only, and thus depends smoothly on the excitation energy $E$, and a number of components with the usual resonant behaviour. It is worthwile mentioning that the resonance parameters $E_{\varrho}, \Gamma_{\varrho}$ and $a_{\varrho}^{R}$ are slightly energy-dependent due to the Greenoperator (16) in the effective hamiltonian (20). Considering a definite resonance $\varrho$ we use resonance parameters fixed at $E=E_{\varrho}$, since the significant contribution of a resonance comes from the energy region around $E_{\varrho}$. If the energy dependence of $\hat{G}_{P}$ is weak and the resonance narrow, the variation of the resonance parameters is negligible, otherwise it describes the deviation from the BreitWigner shape. The occurance of single-particle resonances in the $\mathrm{P}$-space is a special problem which is to be considered separately [9].

The system of equations for the various components of the nuclear state $\left|\Psi_{E, f}^{( \pm)}\right\rangle$can be numerically solved. For that aim one introduces channel states

$$
|c\rangle \equiv\left|E_{c} I_{c} S_{c}, l_{c} j_{c} s_{c} ; J_{c} M_{c}\right\rangle
$$

describing a nucleus with $A$ baryons by the decomposition into a residual nucleus with ( $A$ 1) baryons and excitation energy $E_{c}$, total spin $I_{c}$, strangeness $S_{c}$ and a single separated baryon with angular momentum $l_{c}$, total spin $j_{c}$ and strangeness $s_{c}$. The total spins $I_{c}$ and $j_{c}$ of the two fragments are coupled to the total spin $J_{c}$ with projection $M_{c}$. If additional quantum numbers (e. g. isospin) are necessary to distinguish the various channels they should be included in the definition (37). The dependence on the radial coordinate $r$ of the relative motion between the two fragments is treated explicitely. The channel states $|c\rangle$ form a complete orthonormal basis if all possible combinations of quantum numbers $\mathrm{c}$ are taken into account. In practical calculations, one has to restrict oneself to a few channels. The selection of the important channels is a delicate problem and must be considered in dependence on the reaction parameters and on the structure model for the residual nuclei.

In channel representation, eqs. (18) and (26) represent coupled sets of one-dimensional integro-differential equations with finite kernels. As described in [9] the solutions can be composed of auxiliary functions $F_{c}^{f, R}(r)$ satisfying homogeneous $(f)$ and inhomogeneous $(R)$ sets of differential equations

$$
\begin{aligned}
& {\left[E-E_{c}+\frac{\hbar^{2}}{2 \mu_{c}}\left(\frac{d^{2}}{d r^{2}}-\frac{l_{c}\left(l_{c}+1\right)}{r^{2}}\right)-u_{c}(r)\right] F_{c}^{f, R}(r)-} \\
&-\sum_{c^{\prime}} V_{c c^{\prime}}^{r e s}(r) F_{c^{\prime}}^{f, R}(r)=\left\{\begin{array}{l}
0 \\
\left\langle c|\hat{H}| \Phi_{R}\right\rangle,
\end{array}\right.
\end{aligned}
$$

where $\mu_{c}$ is the reduced mass, $u_{c}(r)$ the average baryon-nucleus potential $(1)$ in channel $c$, and $V_{c c^{\prime}}^{r e s}$ is the matrix element $\left\langle c\left|\hat{V}^{r e s}\right| c^{\prime}\right\rangle$ assuming a zero-range force (9). The latter is responsible for the coupling between the various channels $c$. Knowing $\left|\omega_{E, f}^{( \pm)}\right\rangle$one can calculate the matrix $\hat{M}$ with the elements

$$
M_{R R^{\prime}}=\left\langle\Phi_{R}\left|\hat{H}_{Q Q}^{e f f( \pm)}\right| \Phi_{R^{\prime}}\right\rangle=\left\langle\Phi_{R}|\hat{H}| \Phi_{R^{\prime}}\right\rangle+\left\langle\Phi_{R}\left|\hat{V}^{r e s}\right| \omega_{R^{\prime}}^{( \pm)}\right\rangle
$$

Diagonalizing this matrix one gets the eigenvectors $\left|\Phi_{\varrho}^{(t)}\right\rangle$ and the eigenvalues $E_{\varrho}$ and $\Gamma_{\varrho}$. If one inverts matrix $\hat{M}$ one can finally compose the total nuclear state according to eq. (22). 
Shell-model calculations with a larger number of baryons are extremely time-consuming. The number of degrees of freedom can considerably be reduced if one introduces a modified vacuum state defined by completely occupied proton and neutron shells up to the Fermilevel. Nuclear excitation can then be described by particles above and holes below the Fermilevel. Most of our calculations were performed within a particle-hole approximation with one hyperon and one hole in the closed shells of a magic nucleus. This approach simplifies the calculations essentially but limits the possible application to magic target nuclei. Moreover we have to abandon a detailed description of the hypernuclear fine structure. Simultaneously, the $1 \mathrm{p}-1 \mathrm{~h}$ approach limits the decay channel to residual nuclei which can be described by a one-hole (1h) configuration.

\section{Resonant and quasi-free processes as boundary cases}

In this section, we apply the CSM representation (34) of nuclear and hypernuclear wave functions to the calculation of the production probability of a hypernucleus ${ }_{Y} B$ in a $A(a, b)_{Y} B$ reaction between a target nucleus $A$ and a projectile $a$. Besides energy and momentum the projectile transfers (or produces) strangeness to the target nucleus and is converted into a different particle $b$. Let us assume that the reaction can be described in Born approximation with nuclear wave functions which are independent of the projectile-target interaction. In this case, the reaction probability is determined by the transition matrix element

$$
T_{f i}=\left\langle\Phi_{B} \chi_{b}|\hat{T}| \chi_{a} \Phi_{A}\right\rangle
$$

where initial and final states are described by products of nuclear $\left(\left|\Phi_{A, B}\right\rangle\right)$ and projectile $\left(\left|\chi_{a, b}\right\rangle\right)$ wavefunctions. The most important hypernuclear production reactions can be treated in this manner. Using mesonic projectiles one usually applies the destorted wave impulse approximation (DWIA), which is a Born approximation with mesonic wave functions destorted by the strong projectile-nucleus interaction, while the nuclear wave functions are assumed to be uneffected. In this approximation, the production cross-section $d \sigma / d \Omega$ for a hypernucleus in the state $\left|\Phi_{B}\right\rangle$ can be related to the elementary cross section $(d \sigma / d \Omega)^{\text {elem }}$, which describes the $\mathrm{N}(\mathrm{a}, \mathrm{b}) \mathrm{Y}$ reaction converting a free nucleon $(\mathrm{N})$ into the corresponding hyperon (Y) [10]

$$
\frac{d \sigma}{d \Omega}=\alpha\left(\frac{d \sigma}{d \Omega}\right)^{e l e m} N_{B}^{e f f},
$$

where $\alpha$ is a factor taking the difference between the kinematics of the $A(a, b) B$ and the elementary $\mathrm{N}(\mathrm{a}, \mathrm{b}) \mathrm{Y}$ reaction into account. The nuclear influence on the reaction probability is described by the effective nucleon number $N_{B}^{\text {eff }}$ related to the a definite hypernuclear final state $\left|\Phi_{B}\right\rangle$. It can be written as a nuclear matrix element

$$
N_{B}^{e f f}=\left|\left\langle\Phi_{B}|\hat{W}| \Phi_{A}\right\rangle\right|^{2}
$$

of a transition operator $\hat{W}$ usually approximated by $[11,12]$

$$
\hat{W}=\int d^{3} \vec{r} \chi_{b}^{(-) *}(\vec{r}) \sum_{j=1}^{A} \hat{U}_{j} \delta^{(3)}\left(\vec{r}-\overrightarrow{r_{j}}\right) \chi_{a}^{(+)}(\vec{r}) .
$$

The sum runs over all A nucleons of the target nucleus. The operator $\hat{U}_{\dot{\alpha}}$ converts the $\mathrm{j}$-th nucleon in the corresponding hyperon. In the case of a $n \rightarrow \Lambda$ convertion, $U$ is just the U-spin lowering operator. The sign ( \pm ) means out- and in-asymptotic as explained before formula (14). If one has an unpolarized target and/or does not differ between spin orientations of the hypernucleus, one has additionally to average in eq. (42) over the magnetic quantum numbers of the initial nucleus and to summerize over these numbers in the final hypernucleus. 
The meson wave functions $\chi_{a, b}^{( \pm)}(\vec{r})$ are strongly disturbed by the interaction with the baryons inside the nucleus. We describe this distortion in eikonal approximation [10] with complex phases

$$
\delta_{a}(\vec{r})=\exp \left\{\frac{\bar{\sigma}_{a}}{2}\left(1-i \bar{\alpha}_{a}\right) \int_{-\infty}^{z} \varrho\left(x, y, z^{\prime}\right) d z^{\prime}\right\}
$$

and

$$
\delta_{b}(\vec{r})=\exp \left\{\frac{\bar{\sigma}_{b}}{2}\left(1-i \bar{\alpha}_{b}\right) \int_{z}^{+\infty} \varrho\left(x, y, z^{\prime \prime}\right) d z^{\prime \prime}\right\}
$$

according to

$$
\chi_{a}^{(+)}(\vec{r})=\exp \left\{i \vec{k}_{a} \vec{r}-\delta_{a}\right\} \quad \text { and } \quad \chi_{b}^{(-) *}(\vec{r})=\exp \left\{-i \vec{k}_{b} \vec{r}-\delta_{b}\right\} .
$$

The eikonal phases (44) and (45) are proportional to the corresponding total meson-nucleon cross-sections $\bar{\sigma}_{a, b}$, averaged over the number of protons and nucleons in the target nucleus, to the ratios $\bar{\alpha}_{a, b}$ of the real to the imaginary parts of the corresponding forward scattering amplitudes, and to the effective paths of the mesons through the nucleus. The latter is calculated along a straight line, weighted by the nuclear density $\varrho(r)$, from $-\infty$ to the place $\vec{r}$, where the conversion takes place, and from there up to $+\infty$, respectively. In the numerical calculations, we use experimental values of the meson-nucleon cross-section and neglect the real part of the scattering amplitude $\left(\bar{\alpha}_{a, b}=0\right)$.

If the produced hypernucleus is so highly excited that it can eject a hyperon or a nucleon, the reaction is described by the double differential cross section

$$
\frac{d \sigma}{d \Omega d E}=\alpha\left(\frac{d \sigma}{d \Omega}\right)^{e l e m} \frac{d N_{E f}^{e f f}}{d E}
$$

with a continuous effective nucleon number $d N_{E f}^{e f f} / d E$. This number is a continuous function of the hypernuclear excitation energy $E$ and depends on the decay channel described by the quantum numbers $f$. We get it by replacing the bound hypernuclear final state $\left|\Phi_{B}\right\rangle$ in eq. (42) by the CSM state $\left|\Psi_{E, f}\right\rangle$

$$
\frac{d N_{E f}^{e f f}}{d E}=\left|\left\langle\Psi_{E, f}^{(-)}|\hat{W}| \Phi_{A}\right\rangle\right|^{2}
$$

According to formal scattering theory we have to chose in-asymptotic for the unbound final state. Appart from the effective nucleon number for a particular decay channel $f$ we consider the sum over all open decay channels

$$
\frac{d N_{E}^{e f f}}{d E}=\sum_{f} \frac{d N_{E f}^{e f f}}{d E} .
$$

The effective nucleon number determines the probability of producing a hypernucleus in a given state $\left|\Phi_{B}\right\rangle(42)$ or at a given excitation energy $\mathrm{E}(48)$. It merely differs from the cross section by an (almost) constant factor $\alpha \approx 1$ and by the cross section of the elementary reaction, which can be taken from the experiment. That is why we consider the effective nucleon number instead of the cross section.

If we introduce the CSM representation (34) of the hypernuclear final state into eq. (48) we get a decomposition of the continuous effective nucleon number

$$
\frac{d N_{E f}^{e f f}}{d E}=\left|\left\langle\xi_{E, f}^{(-)}|\hat{W}| \Phi_{A}\right\rangle+\frac{1}{\sqrt{2 \pi}} \sum_{\varrho} \frac{\gamma_{f Q} W_{Q}}{E-E_{g}+i \Gamma_{g} / 2}\right|^{2}
$$

into a coherent sum of 
(i) a term describing the production of the hyperon in an unbound state (quasi-free production), and

(ii) some resonance terms at the energies $E_{\varrho}$ with the widths $\Gamma_{\varrho}$.

The strength of a resonance term is determined by two factors: the matrix element of the interaction operator $\hat{W}$ describing the production of the hypernuclear resonance state $\left|\Omega_{\varrho}\right\rangle$

$$
W_{\varrho}=\left\langle\Omega_{\varrho}^{(-)}|\hat{W}| \Phi_{A}\right\rangle
$$

and the partial width amplitude

$$
\gamma_{f \varrho}=\sqrt{2 \pi}\left\langle\xi_{E, f}^{(-)}\left|\hat{V}^{r e s}\right| \Phi_{\varrho}^{(-) *}\right\rangle
$$

describing the decay of the resonance state $\left|\Phi_{e}\right\rangle$ into the channel $f$.

According to eq. (50) the quasi-free contribution interferes with resonant contributions and the latter interfere among each other. Thus it is not possible, in general, to separate pure quasi-free contributions and pure resonance contributions which can be added up to the total production probability. The hypernuclear production probability does never result from one of the mechanisms (quasi-free or resonant) alone. There are, however, energy regions where one of the various contributions dominates, while the others can be neglected. In such a case, the hypernuclear production probability is dominated by one of the mechanisms. There is, for instance, an upper limit for the resonance energies $E_{\varrho}$ according to the definition of the Q-space. Above this highest resonance energy, the production probability is determined by the quasi-free process and the effective nucleon number is given by

$$
\left(\frac{d N_{E f f}^{e f f}}{d E}\right)_{q . f .}=\left|\left\langle\xi_{E, f}^{(-)}|\hat{W}| \Phi_{A}\right\rangle\right|^{2} .
$$

This quantity describes the production probability per energy unit for a hypernucleus with one baryon in an unbound state. It is a continuous function of the hypernuclear excitation energy E. Since $\hat{W}$ is a single-particle operator, it is only able to transfer a single bound nucleon into an unbound hyperon. That is why hyperon channels are preferably feeded. The possible emission of a nucleon is the consequence of a subsequent action of the residual baryon-baryon interaction $\hat{V}^{r e s}$ mixing hyperon with nucleon decay channels. If both hyperon and nucleon channels are open, hyperon channels dominate usually. However, in dependence on the threshold energies, nucleonic decay channels may be favoured.

Now let us consider the opposite case, that one of the resonance contributions $\varrho$ dominates in eq. (50). If this resonance is sufficiently well isolated from other resonances and the quasifree contribution is negligible within the resonance region around $E=E_{\ell}$, the production probability in channel $f$ is determined by a single resonance term, and we can define the effective nucleon number for a hypernuclear resonance state $\left|\Phi_{\varrho}\right\rangle$

$$
\left(\frac{d N_{E f}^{e f f}}{d E}\right)_{\varrho}=\frac{1}{2 \pi}\left|\frac{\gamma_{f_{\varrho}} W_{\varrho}}{E-E_{\varrho}+i \Gamma_{\varrho} / 2}\right|^{2} .
$$

It describes the production probability of a hypernucleus decaying in channel $f$ via a definite intermediate resonance state $\left|\Phi_{e}\right\rangle$. Again, the resonance state need not necessarily decay into a hyperon channel. Neutron and proton channels can be feeded by the residual baryonbaryon interaction in dependence on resonance structure and threshold energies.

Usually the contribution of a resonance state $\left|\Phi_{\ell}\right\rangle$ is measured by the area below the resonance curve (54) summed over all open decay channels $f$

$$
N_{\varrho}^{e f f}=\sum_{f} \int d E\left(\frac{d N_{E f}^{e f f}}{d E}\right)_{e}=\frac{\sum_{f}\left|\gamma_{f}\right|^{2}}{\Gamma_{e}}\left|W_{g}\right|^{2} .
$$


The last equation has been obtained by neglecting the weak energy dependence of the resonance parameters. Since the main contribution to the integral comes from the energy around $E_{\varrho}$ we have replaced the energy-dependent resonance parameters by their values fixed at $E=E_{\rho}$. This can be done, at least for narrow resonances. Moreover, one can show [13] that, in the case of an isolated resonance and an hermitian hamiltonian, the sum of the partial widths $\sum_{f}\left|\gamma_{f e}\right|^{2}$ equals to the total width $\Gamma_{\varrho}$ and one can ascribe the following effective nucleon number to a definite resonance hypernuclear state $\left|\Phi_{\varrho}\right\rangle$

$$
N_{\varrho}^{e f f}=\left|W_{\varrho}\right|^{2}=\left|\left\langle\Omega_{\varrho}^{(-)}|\hat{W}| \Phi_{A}\right\rangle\right|^{2}=\left|\left\langle\Phi_{\varrho}^{(-)}|\hat{W}| \Phi_{A}\right\rangle+\left\langle\omega_{\varrho}^{(-)}|\hat{W}| \Phi_{A}\right\rangle\right|^{2} .
$$

The approximations leading to eq. (56) are the better fulfilled, the more narrow and isolated the resonance is. Below the lowest hypernuclear disintegration threshold, the hypernuclear production probability is given by eq. (42) with a bound final-state $\left|\Phi_{B}\right\rangle$. Expression (56) coincides with (42) if one identifies $\left|\Phi_{B}\right\rangle$ with $\left|\Phi_{\varrho}^{(-)}\right\rangle$, since the supplement $\left|\omega_{Q}\right\rangle$ vanishes and the sign (-) has no meaning for bound states. Above the first disintegration threshold, the effective nucleon number (56) differs from the expression (41) by the additional term

$$
\left\langle\omega_{\varrho}^{(-)}|\hat{W}| \Phi_{A}\right\rangle=\left\langle\Phi_{\varrho}^{(-)}\left|\hat{V}^{r e s} \hat{G}_{P}^{(-)} \hat{W}\right| \Phi_{A}\right\rangle,
$$

which describes the production of the resonance via continuum and residual interaction. Both resonance production mechanisms interfere, i.e. the resonant process can be either enforced or weakened by the presence of the nuclear continuum. In the general case of overlapping resonances and/or a large quasi-free background, the hypernuclear production can not be parametrized by resonance parameters sufficiently well; instead one has to evaluate the quantity (48) as a continuous function of the hypernuclear excitation energy E. But also in this case, the resonance parameters $E_{\varrho}, \Gamma_{\varrho}$ and $W_{\varrho}$ are usefull parameters, since they help us to determine the energy step of a point-by-point calculation.

Comparing the CSM results with traditional bound-state shell-model calculations one can establish the following influence of the continuous single-particle spectrum:

- there is an additional (quasi-free) term in the hypernuclear production amplitude which describes the production of a hyperon in an unbound scattering state and dominates at large excitation energies,

- above the lowest hypernuclear disintegration threshold, the resonance terms have a finite width due to the coupling between resonance and scattering states, their contributions may overlap and interfere,

- the same coupling causes an additional shift of the resonance energies and modifies resonance wave-functions, production and decay parameters relative to their values in bound-state approximation,

- at any excitation energy, the hypernuclear production probability is a coherent sum of quasi-free and resonant contributions.

There are two main factors governing the influence of the continuous spectrum: the momentum transfer $q$ and the depth $U_{R e}$ of the volume term of the average baryon-nucleus potential defined in eq. (1). The first factor determines the optimal momentum of the produced hyperon in quasi-classic manner.

Fig. 1 illustrates the effect of the different momentum transfers in $\left(K^{-}, \pi^{-}\right)$strangeness transfer at (almost) magic kaon momentum and $\left(\pi^{+}, K^{+}\right)$associated strangeness production. In the first case, the momentum transfer is low $(q \approx 50 \mathrm{MeV} / \mathrm{c})$ and the production of hypernuclear states around the disintegration threshold is favoured. Preferably substitutional states are populated, while the hypernuclear ground state is almost not excited. The momentum transfer of the strangeness production is essentially larger $(q \approx 300 \mathrm{MeV} / \mathrm{c})$ and 


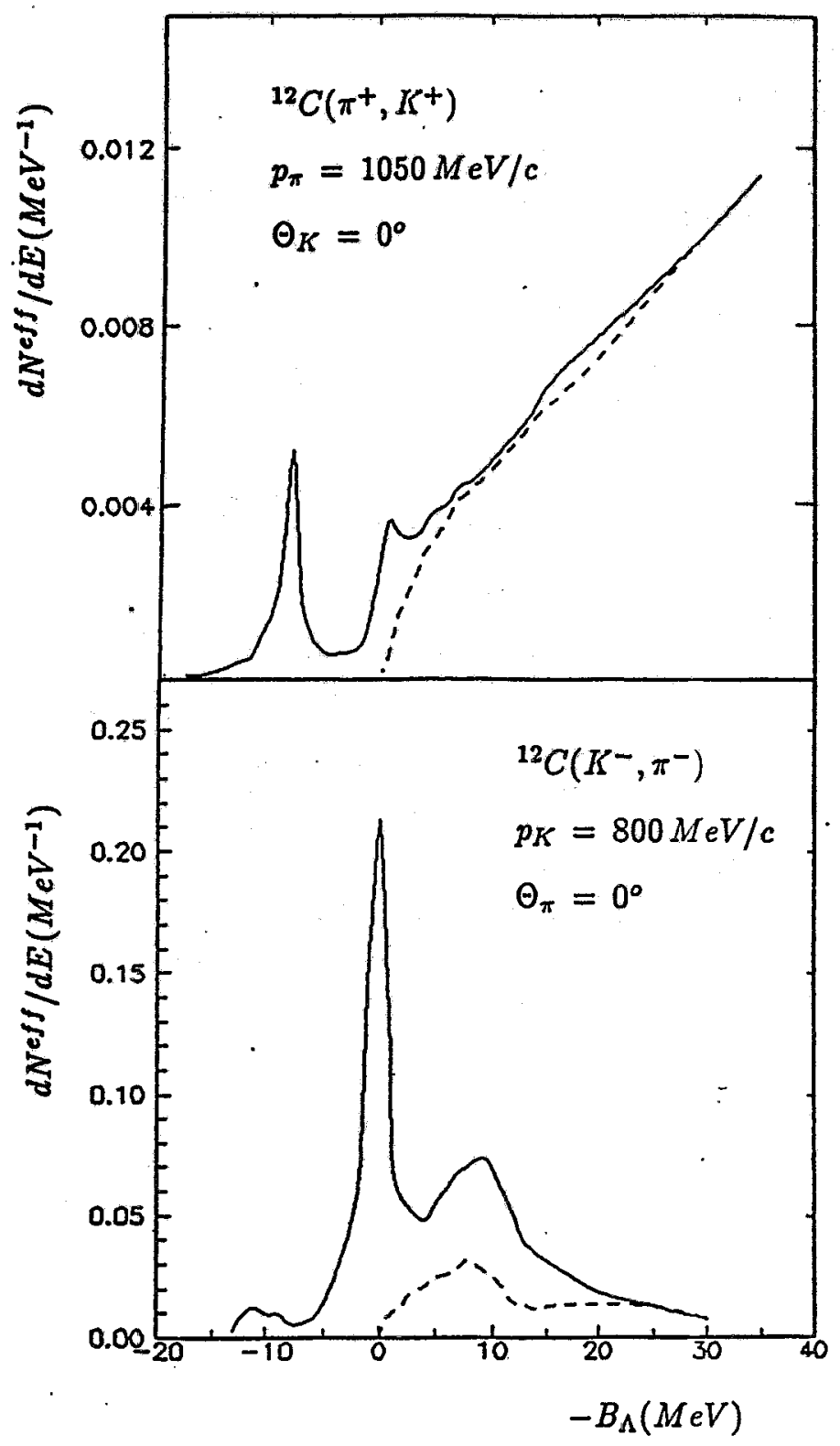

Fig. 1: Continuous effective nucleon number $d N_{E}^{e f f} / d E$ for $\Lambda$ hypernuclear production in collinear geometry $\left(\Theta_{\pi, K}=0^{\circ}\right)$ calculated in $1 p-1 h$ CSM and DWIA for strangeness transfer (lower part) and for associated strangeness production (upper part). The hypernuclear excitation energy is expressed by the negative hyperon binding energy $-B_{\Lambda}$ The broken lines show the quasi-free contributions defined by eq. (53), while the full line corresponds to the coherent total sum (50). Bound and resonance states have been spread over a $2 \mathrm{MeV}$ Breit-Wigner distribution. The curves show the sum over all open channels $\mathrm{f}$ which have been taken into account.

the quasi-free process dominates. Its maximum is around $-B_{A}=45 \mathrm{MeV}$. Below the threshold, the ground state is well pronounced, while the resonance structures above the threshold vanish in the quasi-free background. The absolute scales on the vertical axis indicate that strangeness transfer is more probably than production. 


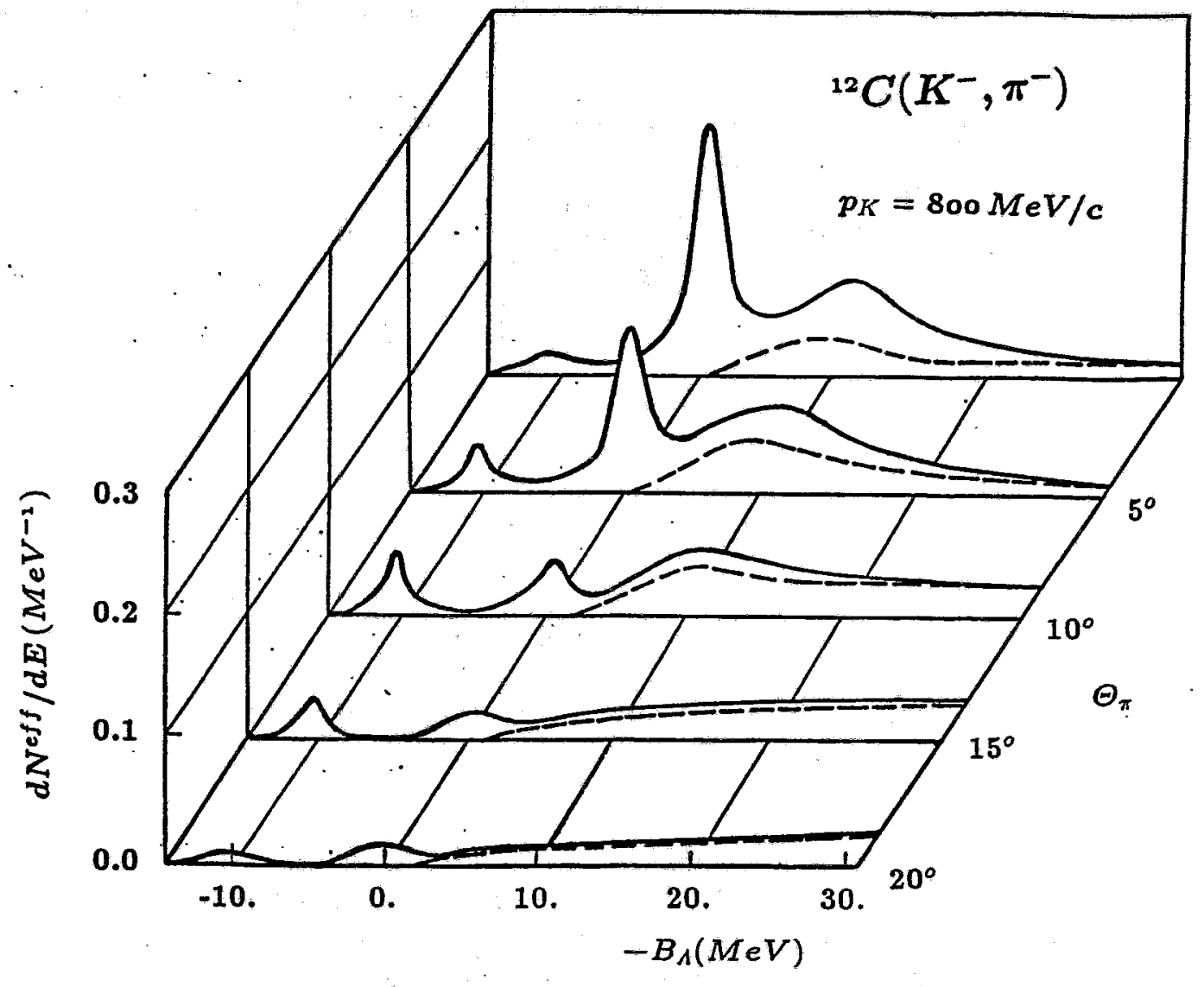

$0^{\circ}$

Fig. 2: The same as in fig. 1 for the ${ }^{12} C\left(K^{-}, \pi^{-}\right)$strangeness transfer at different scattering angles $\Theta_{\pi}$. The contributions of bound and resonance states have additionally been spread by a $3 \mathrm{MeV}$ Breit-Wigner distribution.

We use the following parameters of the average $\Lambda$-nucleus potential and of the $\Lambda$-nucleon residual interaction with the notation of eqs. (1) to (4) and (9), respectively

$$
\begin{array}{rlrl}
U_{R e}^{\Lambda}=28 \mathrm{MeV} & U_{\text {s.o. }}^{\Lambda}=2 \mathrm{MeV} \\
r_{o}=1.1 \mathrm{fm} & d=0.6 \mathrm{fm} .
\end{array}
$$

The average potential is compatible with a residual $\Lambda N$ contact interaction with the parameters

$$
V_{a}^{r e s}=300 \mathrm{MeVfm}^{3} \quad a=1.05 \quad b=-0.1 .
$$

The parameters of the average nucleon-nucleus potential are determined by the experimental values of the corresponding single-particle thresholds.

The dependence of the production probability on the angle $\Theta$ between the projectile beam axis and the direction of mesured meson (afterwards denoted as "scattering angle") is illustrated in Fig. 2. Here one sees the continous transformation from the dominating "recoilles" production of substitutional states at $\Theta_{\pi}=0^{\circ}$ with small background and ground-state excitation to a dominating quasi-free process at $\Theta_{\pi}=20^{\circ}$. Fig. 3 shows the contributions of individual hypernuclear bound and resonance states and of particular decay channels $f$ to the assiciated hypernuclear production on ${ }^{28} \mathrm{Si}$. Due to the $1 \mathrm{p}-1 \mathrm{~h}$ approach the decay modes are 
restricted to $1 \mathrm{~h}$ states of ${ }^{27} \mathrm{Si}$. At larger excitation energies, the contributions of the various decay channels correspond to their statistical weight determined by the spin of the residual nucleus.

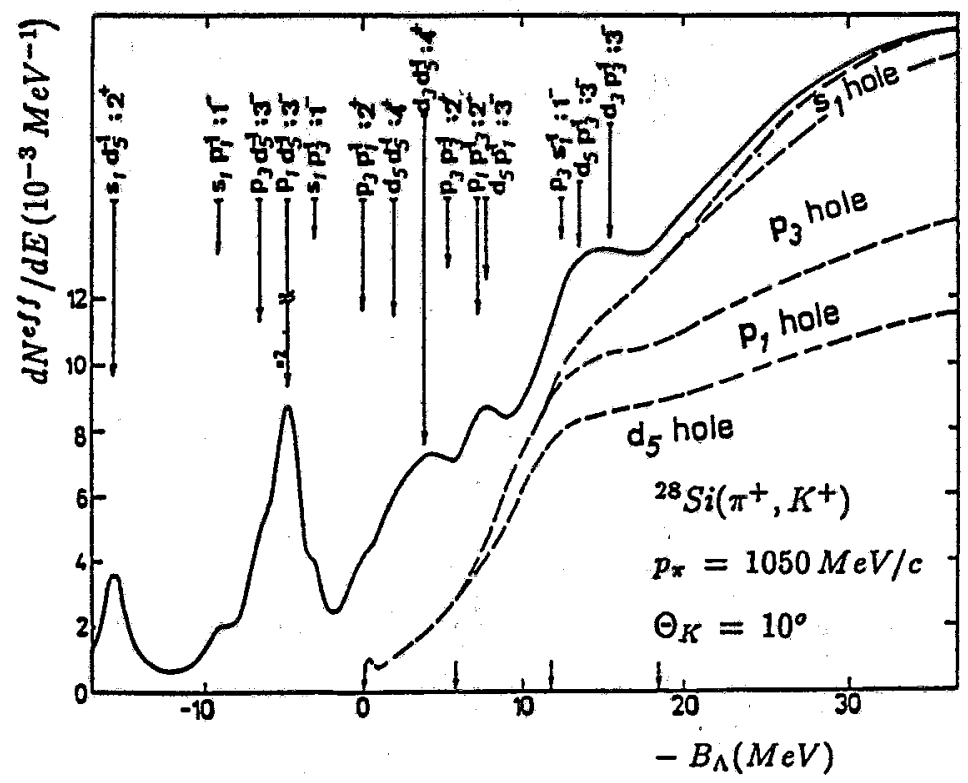

Fig. 3: The same as in fig. 1 for the ${ }^{28} \mathrm{Si}\left(\pi^{+}, K^{+}\right)$associated strangeness production. Additionally to the continuous effective nucleon number the contributions $N_{\varrho}^{\text {eff }}$ (56) of individual hypernuclear bound and resonance states with $1 p-1 \mathrm{~h}$ structure $\left(l_{j}\right)_{\Lambda}\left(l_{j}\right)_{n}^{-1} ; J$ are indicated by arrows above the resonance peaks. The notations are used as $d_{5}$ for $1 d_{5 / 2}$ etc. The contributions of bound and resonance states to the solid curve have additionally been spread by a $3 \mathrm{MeV}$ Breit-Wigner distribution. The broken lines give the partial contributions of the indicated decay channles. Their thresholds are marked by the small arrows at the $-B_{\Lambda}$-axis.

\section{Consequences of the shallow hyperon-nucleus potential}

In the course of our investigations of $\Lambda$ and $\Sigma$ hypernuclei we noticed a crucial dependence of the hypernuclear production process on the strength of the average hyperon-nucleus potential. Hyperons are generally more weakly bound in nuclei than nucleons. For $\Lambda$ hyperons a value $U_{R e}=28 \pm 2 \mathrm{MeV}$ has been established. The situation for $\Sigma$ hyperons continues to be unclear. A value around or below $20 \mathrm{MeV}$ seems to be favoured. A depth of $12 \mathrm{MeV}$ was deduced from a presumable $\frac{4}{\Sigma} \mathrm{He}$ ground state. Analysing corresponding decay processes the average depth for cascade particles $\Xi$ in nuclei was determined to be between 21 and $24 \mathrm{MeV}$. The small depth of the average potential has several important consequences for the hypernuclear spectrum and production probability. Since, in a shallower potential, the number of bound hyperon single-particle shells is smaller, there are less bound and resonance states in the hypernuclear excitation sprectra. The importance of the quasi-free mechanism increases correspondingly. In comparison to a nuclear excitation reaction (e. g. (e,e')) at the same momentum transfer, the quasi-free contribution to a hypernuclear production reaction is larger.

Weakly bound single-particle hyperon states play an important role. Fig. 4 displays the single-particle energy for a $\Sigma$ hyperon in a $A=15$ nucleus for a large variety of potential parameters. One sees that it is very likely to find single-particle levels in the close neigbourhood of the threshold at $B_{\Sigma}=0$, in particular for negatively charged hyperons. The energy of 


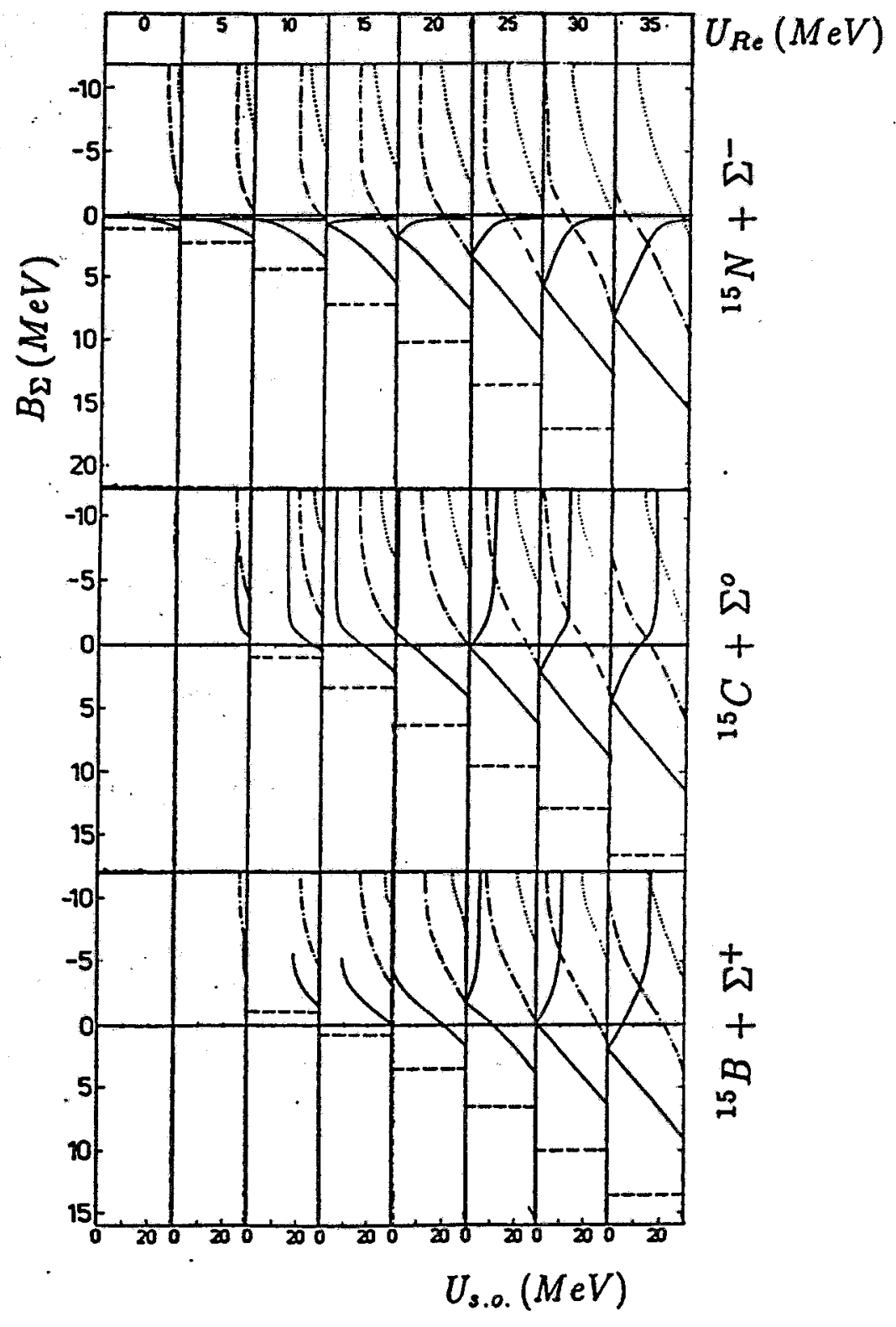

Fig. 4: Single-particle spectra of $\Sigma^{-}, \Sigma^{\circ}$ and $\Sigma^{+}$in $A=16, Z=6$ hypernuclei for varying potential depths $U_{R e}$ and $U_{\text {s.o. }}$ (1). (full curves: $1 p_{3 / 2}$ and $1 p_{1 / 2}$; dashed curves: $1 s_{1 / 2}$; dashdotted curves: $1 d_{5 / 2} ;$ dotted curves: $1 f_{7 / 2}$ ).

Negative binding energies are defined by the scattering phase $\delta_{l j}=\pi / 2$.

such states is not proportional to the strength of the spin-orbit potential as assumed in the oscillator model. It is almost independent on it. Weakly bound states distinguish themselfes by a large mean square radius. Their radial wave functions deviate clearly from the corresponding harmonic oscillator wave functions. They are essentially different from the deeper bound nucleon wave functions with the same quantum numbers. The difference between proton and $\Sigma^{-}$radial wave functions is illustrated in fig. 5 . The crucial parameter is the ratio $\varepsilon_{\Sigma} / \varepsilon_{p}$ of the hyperon to proton binding energy. The difference between the hyperon and nucleon wave functions is quantified by the deviation of the overlap integral

$$
R=\left|\int d r \varphi_{Y}(r) \varphi_{N}(r)\right|^{2} .
$$


from unity. Fig. 6 shows the dependence of $R$ on the hyperon binding-energy, while the nucleon has kept fixed and deeply bound. A remarkable deviation from unity appears for hyperons which are more loosely bound than $2 \mathrm{MeV}$.

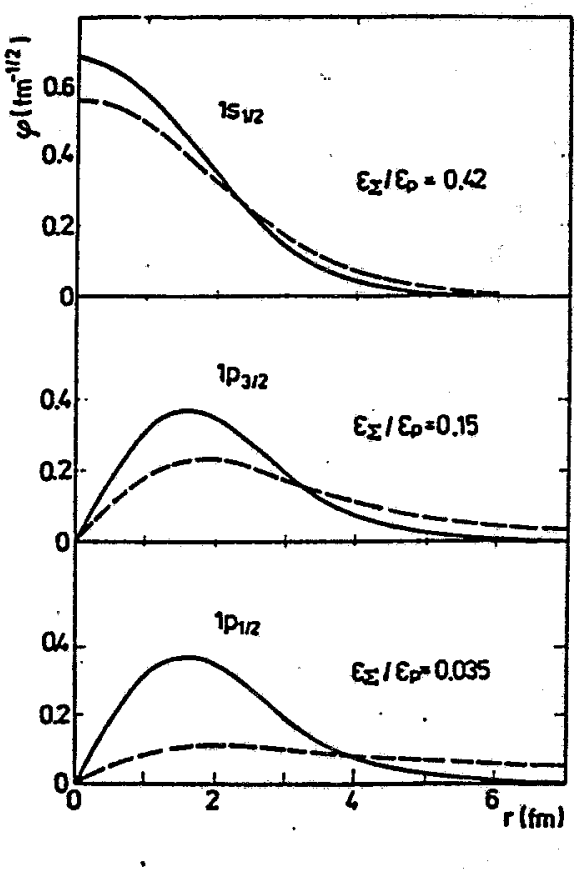

Fig. 5: Comparison between radial functions of deeply bound protons and weakly bound $\Sigma^{-}$hyperons for three values of the $\Sigma^{-}$to proton binding energies.

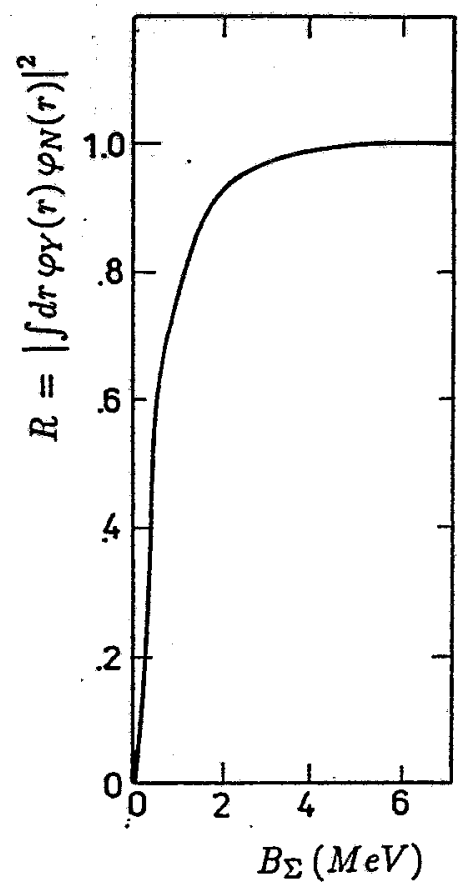

Fig. 6: Overlap integral $R=$ $\left|\int d r \varphi_{\Sigma}(r) \varphi_{p}(r)\right|^{2}$ between a weakly bound hyperon and a deeply bound proton radial wave function as a function of the $\Sigma^{-}$binding energy $B_{\Sigma}$.

The integral (61) determines the recoilless production probability of substitutional hypernuclear states in plane wave approximation. According to fig. 6 the production of hyperons in weakly bound states is obstructed. It tends to zero if the hyperon state approaches to the threshold at $B_{Y}=0$. In particular the population of Coulomb assisted hypernuclear states is affected. Meson distortion and a small momentum transfer moderate this effect only a little.

The consequences of this effect are illustrated in fig. 7, where we tried to reproduce a $\Sigma$ hypernuclear excitation spectrum with a double hump structure seemingly observed in the experiment [14]. To reproduce two peaks corresponding to $p_{1 / 2}$ and $p_{3 / 2}$ substitutional states one needs a spin-orbit splitting of p-shell $\Sigma$ hyperons of $12 \mathrm{MeV}$. Such a huge splitting is incompatible with an avarage potential shallower than $U_{R e} \approx 25 \mathrm{MeV}$ (see fig. 4). To get a $12 \mathrm{MeV}$ splitting with a sufficiently well bound, and thus well populated $\mathrm{p}_{1 / 2}$ shell, one needs at least $\mathrm{U}_{R e}=30 \mathrm{MeV}$ and $\mathrm{U}_{\text {s.o. }}=20 \mathrm{MeV}$ (upper part of fig. 7). The bump resulting from the $p_{1 / 2} p_{1 / 2}^{-1}$ substitutional state is then still enlarged by a broad $s_{1 / 2} s_{1 / 2}^{-1}$ substitutional and a $d_{5 / 2} p_{3 / 2}^{-1}$ non-substitutional state almost at the same energy. However, a potential depth of $30 \mathrm{MeV}$ is not in agreement with the $\frac{4}{\Sigma} \mathrm{He}$ ground-state energy. 


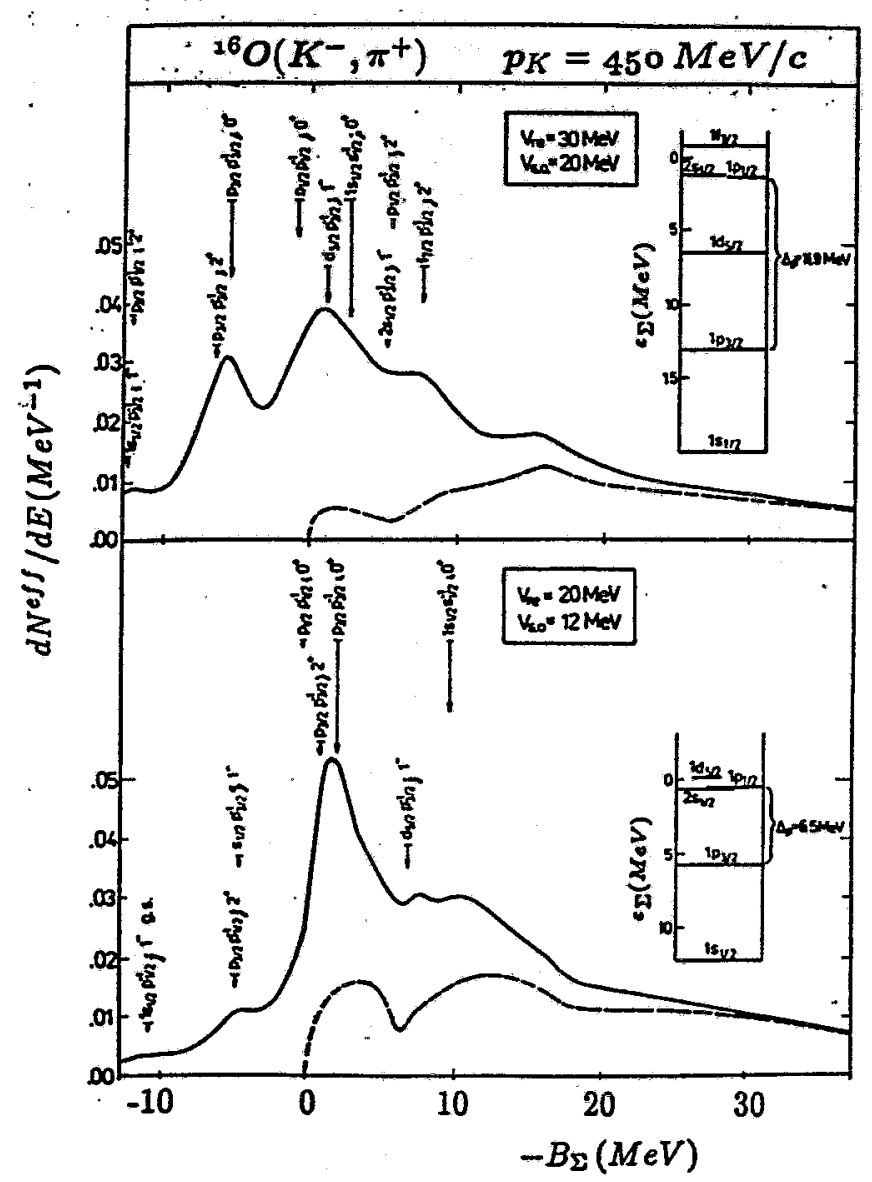

Fig. 7: Continuous effective nucleon number $d N_{E}^{e f f} / d E$ for $\left(K^{-}, \pi^{+}\right) \Sigma$ hypernuclear production at $p_{K}=450 \mathrm{MeV} / \mathrm{c}$ in collinear geometry $\left(\Theta_{\pi}=0^{\circ}\right)$ calculated in 1p-1h CSM and DWIA for two sets of the average hyperon-nucleus potential. The hypernuclear excitation energy is expressed by the negative hyperon binding energy $-B_{\Sigma}$ The broken lines show the quasi-free contributions defined by eq. (53), while the full line corresponds to the coherent total sum (50). Bound and resonance states have been spread over a $3 \mathrm{MeV}$ Breit-Wigner distribution. The curves show the sum over all open channels $\mathrm{f}$ which have been taken into account. The $\Sigma^{-}$single-particle levels are displayed in the inserts. The arrows above the curves indicate the positions of resonance peaks with a definite $1 p$-1h structure. The lengths of the arrows correspond to the strengths of the resonances defined by expression (56).

Further decreasing the potential depth $U_{R e}$ the $p_{1 / 2}$ shell comes inescapably closer to the threshold and its population probability tends to vanish. Moreover, the $p_{1 / 2} p_{1 / 2}^{-1}$ peak is shifted towards the $p_{3 / 2} p_{3 / 2}^{-1}$ peak, even if the depth of the spin-orbit potential would be kept constant. There is already no second substitutional bump at $U_{R e}=20 \mathrm{MeV}$ and $V_{3 . o .}=12 \mathrm{MeV}$ (lower part of fig. 7 ). The $d_{5 / 2}$ shell is unbound and gives rise to a very small bump only. Altogether we were unable to find a parameter set with $U_{R e} \approx 20 \mathrm{MeV}$ or shallower which would reproduce two distinct resonance peaks. On the other hand we noticed that the quasi-free contribution gets a resonance-like structure when decreasing the potential depth (compare the broken lines in both part of fig. 7). We investigated this effect in fig. 8 in detail, where we have further decreased the potential depth. 


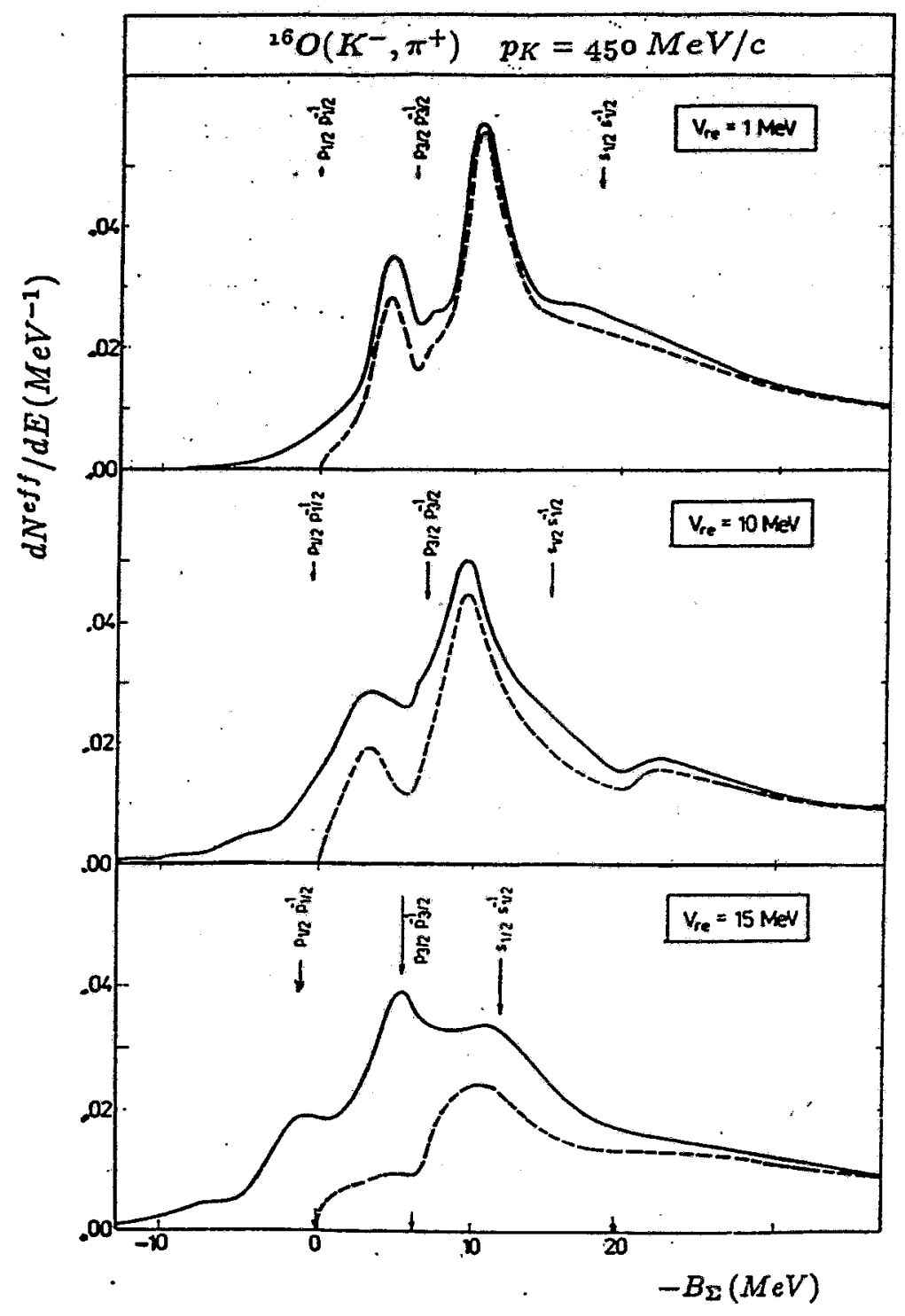

Fig. 8: The same as in fig. 7 for 3 shallower potentials and vanishing spin-orbit coupling. The arrows at the $-B_{\Sigma}$-axis indicate the thresholds for $\Sigma^{-}$emission with a residal nucleus described by a $p_{1 / 2}^{-1}, p_{3 / 2}^{-1}$ and $s_{1 / 2}^{-1}$ ih state, respectively.

We observed a transformation of the calculated hypernuclear production probability expressed by the continuous effective nucleon number. While, for potentials deeper than $15 \mathrm{MeV}$, resonance states with a definite $1 \mathrm{p}-1 \mathrm{~h}$ structure dominate the spectrum, threshold effects determine the energy dependence for shallower potentials. A resonance-like peak occurs approximately $3 \mathrm{MeV}$ above the emission thresholds for hyperons. The distance between these peaks is determined by the excitation energies of the corresponding residual nuclei. In the case of fig. 8 , the distance between the two main peaks is given by the energy of the ${ }^{15} \mathrm{~N}$ excited $3 / 2^{-}$state lying $6.2 \mathrm{MeV}$ above the ground state. This distance is a property of the residual nucleus which consists of nucleons only. Hence the bump distance is unsuitable for extracting any information about the hyperon-nucleus or hyperon-nucleon interaction. Hypernuclear resonance states, which might yield such informations are too weakly populated and buried under the steep threshold behaviour. So we have to conclude that it is very difficult to deduce hyperon interaction parameters from a hypernuclear exci- 
tation spectrum if the hyperon-nucleus potential is weaker than $\approx 15 \ldots 20 \mathrm{MeV}$, even in the case of an optimal chosen momentum transfer.

A potential depth of $17 \mathrm{MeV}$, as deduced in [7], is already incompatible with a strong spinorbit splitting. In ref. [15], we considered the effective nucleon number for the ${ }^{12} C\left(K^{-}, \pi^{+}\right)$ and ${ }^{16} O\left(K^{-}, \pi^{+}\right)$strangeness and charge transfer reactions at $p_{K}=450 \mathrm{MeV} / \mathrm{c}$. We showed that the energy dependence is clearly determinated by the threshold behaviour of the quasifree process, if the average hyperon-nucleus potential is $15 \mathrm{MeV}$ deep. In this case, it is almost independent of the strength of the spin-orbit potential and not sensitive to a further decrease of the potential $U_{R e}$. The $p_{1 / 2}$ hyperon in ${ }_{\Sigma}^{16} \mathrm{O}$ does not produce a noticable bump, since it is too loosely bound.

The appearance of threshold effects gives an alternative explanation of the observed double-hump structure in the $\left(K^{-}, \pi^{+}\right)$spectrum on ${ }^{16} \mathrm{O}$. Moreover the threshold effects are generated by $\Sigma$ hyperons leaving the nucleus within a time which is comparable with the strong interaction time. Hence they should be less influenced by the strong $\Sigma^{-} p \rightarrow \Sigma^{o} n$ and $\Sigma^{-} p \rightarrow \Lambda \Lambda$ conversion processes, which has not been taken into account in our calculations. Long-living $\Sigma$ hypernuclear resonance states however should get an additional large width due to the conversion process in contrast to the experimental observation.

\section{Baryonic decay channels of $\Lambda$ hypernuclei}

Most of the hypernuclei produced in strangeness-transfer or production reactions are highly excited and can decay by emitting a hyperon, a nucleon or a cluster of baryons. This decay is a property of the (excited) hypernuclear many-body system and is caused by the strong interaction with the assistance of the electrostatic force. It is aquivalent to the usual nuclear decay by neutron, proton and $\alpha$ cluster emission or fission and must not be confused with the weak decay of the hyperon itself. Forecasts of branching ratios between various strong disintegration or electromagnetic deexcitation channels are desired since it got possible to trace experimentally not only the mere production of a hypernucleus, but to detect $\gamma$ quanta emitted from excited hypernuclei and even $\gamma$ quanta accompanying baryon emission. The latter allows us to select definite decay channels. The description of the decay of hypernuclei encompasses details of the structure of the parent and daughter hypernuclei (or nuclei) and thus, many theoretical assumptions may be sensitively verified in hypernuclear decays. Moreover, the production of a hypernuclear resonance state and its subsequent decay onto a daughter level by nucleon emission may yield hypernuclear states which would not be obtained otherwise.

Analysis of hypernuclear resonance decays in the framework of the bound shell-model with oscillator basis in combination with the R-matrix theory appeared to be a reasonable description of many-body aspects, such as symmetries of the wave functions [16]. In some cases, decay channels are appreciably suppressed for symmetry reasons, which are otherwise energetically open. In such a way, the appearence of $\gamma$ deexcitation quanta of several $\mathrm{MeV}$ energy may be explained. R-matrix theory, however, is an approximation and not applicable to broad resonances with a lifetime in the order of the strong interaction time. Moreover, the oscillator basis does not take the importance of weakly bound hyperon states into account.

The emission of a baryon from a quasi-bound hypernucleus state can easily be understood and described by the CSM frame developed in sections 2 and 3 . Sufficiently narrow resonances are described by the parameters $E_{\ell}, \Gamma_{\ell}(28,30)$ and $W_{\ell}(51)$ calculated at the energy $\mathrm{E}=\mathrm{E}_{\varrho}$. Partial widths $\Gamma_{f \varrho}$ of a resonance $\varrho$ for the decay into channel $f$ are given by

$$
\Gamma_{f g}=\left|\gamma_{f e}\right|^{2} .
$$

The relation between different decay channel is described by the relative partial decay width 


$$
\mathcal{R}_{f e}=\frac{\Gamma_{f e}}{\sum_{f} \Gamma_{f \varrho}} .
$$

For broader resonances, the energy dependence of these parameters gets important. They should rather be described by the continuous function $d N_{E f}^{e f f} / d E$ (48). The population of the various $1 \mathrm{~h}$ channels in the ${ }^{28} \mathrm{Si}\left(\pi^{+}, \mathrm{K}^{+}\right)_{\Lambda}^{28} \mathrm{Si}$ reaction followed by $\Lambda$ emission has already been investigated in fig. 3. Due to the large momentum transfer, the $\Lambda$ channels dominate and we could neglect other decay modes.

If one want to compare hyperon and nucleon decay channels one has to go beyond the $1 \mathrm{p}-1 \mathrm{~h}$ approximation. To tackle such problems we coupled a bound-state shell-model with oscillator basis for the description of complicated residual nuclei to our continuum shellmodel. (The emission of more than one baryon remains beyond the scope of our model.) We used the weak coupling basis with a single particle (hyperon or nucleon) coupled to a residual nucleus $\left|t_{c}\right\rangle$, which consists of (A-1) baryons. The selected baryon may be either in the discrete or in the continuous part of the spectrum. Instead of a simple hole the residual nucleus is described by a complicated shell-model wave function calculated within a translationally invariant bound-state shell-model [17]. Restricting to p-shell nuclei we used the central nucleon-nucleon residual interaction of Boyarkina [18]. The $\Lambda N$ interaction was approximated by a contact force (9) with the parameters (60). The use of the harmonic oscillator basis for the description of the residual nuclei is a certain inconsistency in our approach, but it simplifies the model considerably and allows us to apply techniques particularly developed for that basis, e. g. a method for excluding center-of-mass motion. Usually details of the hypernuclear and nuclear structure are more important for the properties of bound and quasi-bound states than a consistent description of the coupling with the scattering states. That is why we prefered a more sophisticated structure model for the description of resonant processes, while we used simple $1 \mathrm{p}-1 \mathrm{~h}$ and $1 \mathrm{~h}$ structures for the quasi-free process.

Figs. 9 and 10 show two decay schemas of hypernuclear resonance states. We have displayed only those states which are preferentially populated in a strangeness transfer reaction at small momentum transfer. The proton rich hypernucleus ${ }_{\Lambda}^{14} \mathrm{~N}$ is an example of a favoured nucleon emission. The uppermost state with a dominating $p_{3 / 2}^{\Lambda} \otimes \frac{5}{2}^{-}$configuration is the most probably populated state. It exhausts $53 \%$ of the total hypernuclear production probability and decays mainly via $\Lambda$ emission to the ground state of ${ }^{13} \mathrm{~N}$. Nonetheless $20 \%$ go via proton emission to excited low lying states of ${ }_{\Lambda}^{13} \mathrm{C}$. They are de-excited by a secondary $\gamma$ emission in turn.

The state with the dominating $p_{1 / 2}^{\Lambda} \otimes \frac{1}{2}^{-}$component, which is not very much populated by the recoilless strangeness transfer, can decay exclusively to the daughter hypernucleus ${ }_{\Lambda}^{13} \mathrm{C}$, preferably to its ground state. The excited $p_{3 / 2}^{\Lambda} \otimes \frac{3}{2}^{-}$state (still more weakly excited) feeds mainly the excited ${ }_{\Lambda}^{13} \mathrm{C}$ hypernuclear states. The comparison with a pure bound-state shellmodel calculation [19] shows that the excitation strengths of the important hypernuclear resonance states are practically unchanged by the inclusion of the continuum. In CSM, the branching ratios for proton emission are slightly increased as opposed to hyperon ones.

Investigating the decay modes of ${ }_{\Lambda}^{14} \mathrm{C}$ (fig. 10 ) we included a strongly populated state with isospin $\mathrm{T}=3 / 2$ and dominating $p_{3 / 2}^{\Lambda} \otimes\left(\frac{3}{2}^{-}, \frac{3}{2}\right)$ structure [20]. Although allowed energetically, the $\Lambda$ emission is strongly suppressed by isospin conservation. Hypernuclear doublets of ${ }_{\Lambda}^{13} \mathbb{B}$ and ${ }_{\Lambda}^{13} \mathrm{C}$ are feeded instead. 


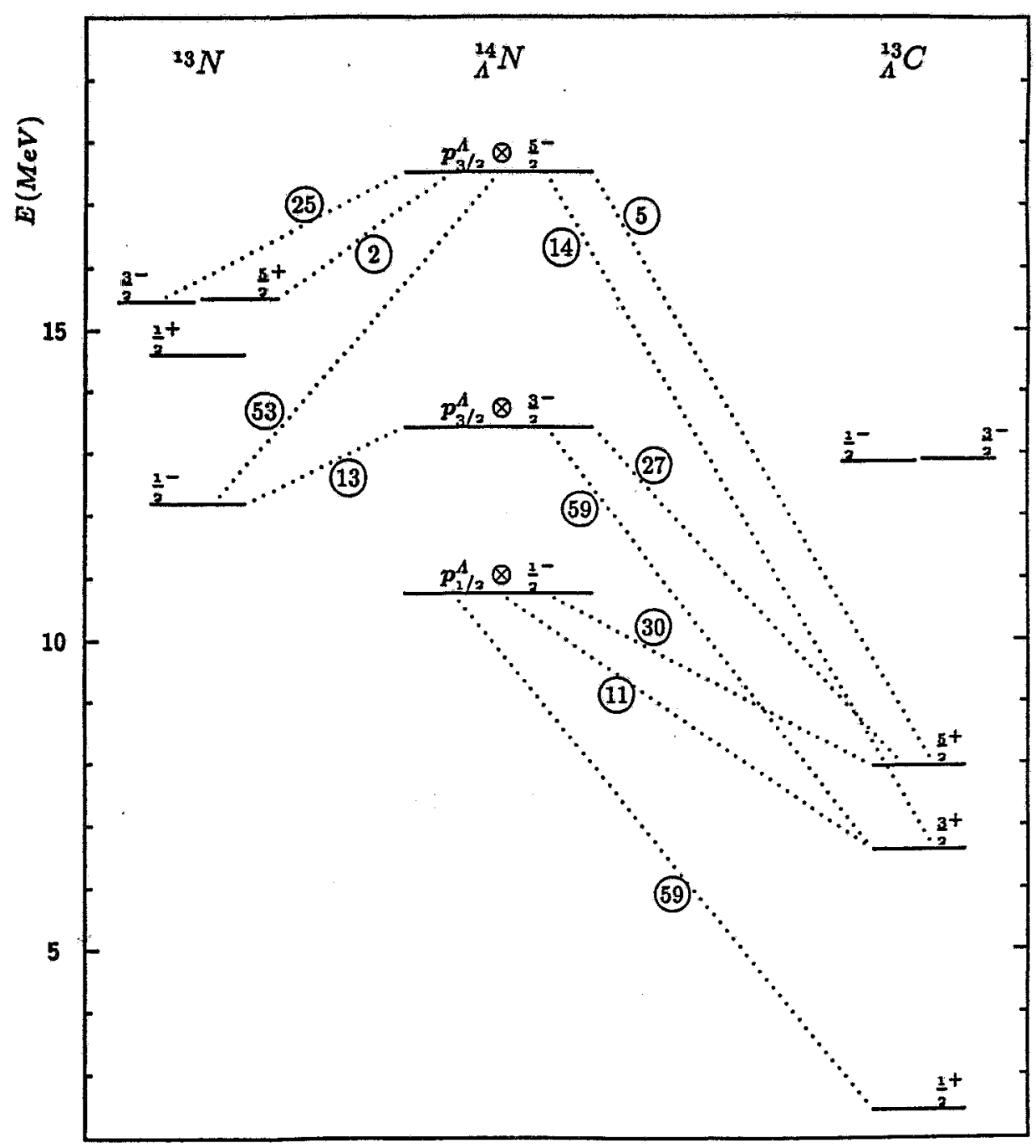

Fig. 9: Decay schema with calculated branching ratios $\mathcal{R}_{f \varrho}$ (in percent) of those $1^{+}$resonances of ${ }_{\Lambda}^{14} N$ which are predominantly excited in ${ }^{14} N\left(K^{-}, \pi^{-}\right)$ strangeness transfer at vanishing momentum transfer. The resonance states are characterized by their main component in the particle $\otimes$ core coupling schema. Only $T=1 / 2$ core states have been taken into account.

Decay considerations may serve as a reasonable basis for the preparation of new experiments. Especially a purposeful decay population of excited hypernuclear doublets and their subsequent $\gamma$ de-excitation (here $\frac{3}{2}^{+}$and $\frac{5}{2}^{+}$) is worthwile and may be used in distinguishing various models of hyperon-nucleon interactions. 


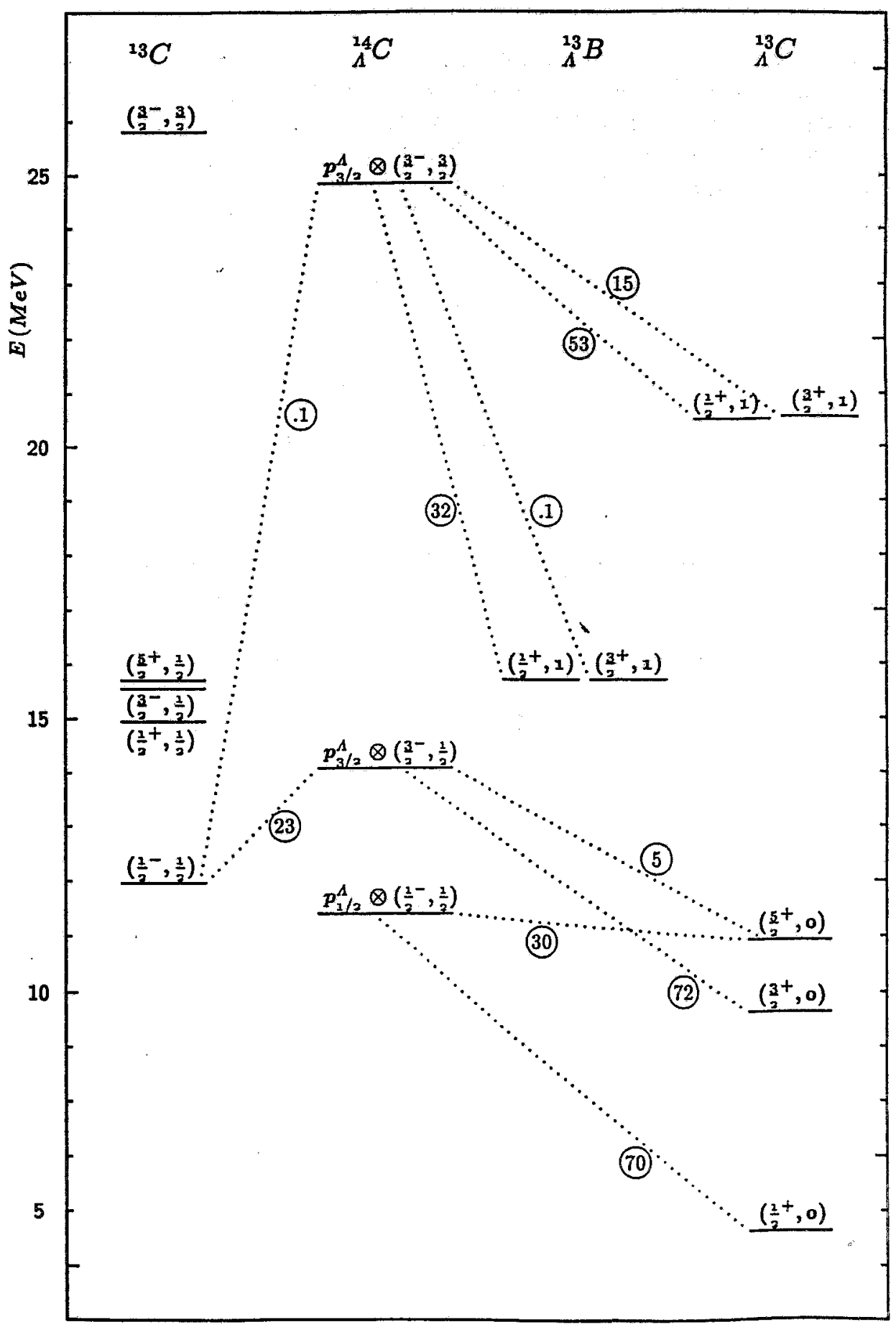

Fig. 10: Decay schema with calculated branching ratios $\mathcal{R}_{f} \varrho$ (in percent) of the $\mathrm{O}^{+}$substitutional states of ${ }_{\Lambda}^{14} \mathrm{C}$. The resonances are characterized by their main component in the particle core coupling schema. Core states are distinguished by their spin and isospin $(J, T)$. 


\section{Conclusions}

It has been the aim of this paper to sketch the merits of an unified description of resonant and quasi-free processes of hypernuclear production which are otherwise treated separately. In numerical calculations, we have demonstrated the relation between both mechanisms in dependence on the kinematic parameters and on the binding energy of the hyperon in the produced hypernucleus. It has turned out that, due to the shallow hyperon-nucleus potential, a simultaneous consideration of bound and unbound hyperon states is essential in most cases of hypernuclear production.

We have offered an alternative interpretation of the bump structure observed in the $\Sigma$ hypernuclear production cross-section. According to that it is practically impossible to determine the parameters of the hyperon-nucleon interaction by means of a measured production cross-section if the average hyperon-nucleus potential turns out to be weaker than $20 \mathrm{MeV}$. Considering disintegration processes of long-lived $\Lambda$ hypernuclear states we have established agreement with calculations within a bound-state shell-model in principle.

\section{Acknowledgement}

The work reported above is the result of a continuous collaboration with J. Z̆ofka and L. Majling for several years. I am indebted to them for all the collaborations, suggestions and discussions which underly the present paper. The author is also grateful to the Nuclear Physics Institute ( $\check{R} e \check{z}$ ) for the warm hospitality extended to him several times in the course of this work.

The paper was partially supported by the BMFT Bonn (contract 06 DR 107).

\section{References}

[1] Dalitz, R.H. and A. Gal

Ann. Phys. (N.Y.) 116 (1978) 167

[2] Auerbach, E.H., A.J. Baltz, C.B. Dover, A. Gal, S.H. Kahana, L. Ludeking and D.J. Millener

Ann. Phys. (N.Y.) 148 (1983) 381

[3] Majling, L., J. Žofka, V.N. Fetisov and R.A. Eramzhyan

Nucl. Phys. A450 (1986) 189c; Phys. Lett. 183B (1987) 263; 202B (1988) 489

[4] Halderson, D., Y. Mo and P. Ning

Phys. Rev. Lett. 57 (1986) 1117

[5] Morimatsu, O. and K. Yazaki

Nucl. Phys. A435 (1985) 727

[6] Kishimoto, T.

Nucl. Phys. A450 (1986) 445c

[7] Hausmann, R. and W. Weise

Z. Phys. A324 (1986) 355

[8] Motoba, T., H. Bando, R. Wünsch and J. Žofka

Phys. Rev. C38 (1988) 1322

[9] Barz, H.W., I. Rotter and J. Höhm

Nucl. Phys. A275 (1977) 111 
[10] Dover, C.B., L. Ludeking and G.E. Walker

Phys. Rev. C22 (1980) 2073

[11] Dalitz, R.H. and A. Gal

Phys. Lett 64B (1976) 154

[12] Boussy, A.

Phys. Lett 99B (1981) 373

[13] Mahaux, C. and H.A. Weidenmüller

Shell-model approach to nuclear reactions (North-Holland, Amsterdam, 1969)

[14] Bertini, R. et al.,

Phys. Lett. 90B (1980) 375; 136B (1984) 29; 158B (1985) 19

[15] Wünsch, R. and J. Žofka

Phys. Lett. B193 (1987) 7

[16] Majling, L., J. Žofka, V.N. Fetisov and R.A. Eramzhyan

Proc. of the 1986 INS Int. Symp. on Hypernuclear physics (Tokyo 1986)

eds. H. Band $\bar{o}$, O. Hashimoto and K. Ogawa, p. 112

[17] Majling, L. et al.

Phys. Lett. 130B (1983) 235

[18] Balashov, V.V., A.N. Boyarkina, and I. Rotter Nucl. Phys. 59 (1964) 417

[19] Wünsch, R., L. Majling and J. Z̆ofka Nucl. Phys. A450 (1986) 329c

[20] Wünsch, R., L. Majling and J. Z̆ofka Czech. J. Phys. B27 (1986) 441 